Old Dominion University

ODU Digital Commons

2012

\title{
Haptics-Augmented Training Software for Undergraduate Engineering Mechanics
}

\author{
Ernur Karadogan \\ Robert L. Williams \\ David R. Moore \\ Tian Luo \\ Old Dominion University
}

Follow this and additional works at: https://digitalcommons.odu.edu/stemps_fac_pubs

Part of the Curriculum and Instruction Commons, and the Social Media Commons

\section{Original Publication Citation}

Karadogan, E., Williams, I. I. R. L., Moore, D. R., \& Luo, T. (2012). Haptics-augmented training software for undergraduate engineering mechanics. (45035), 1007-1014. doi: 10.1115/DETC2012-70250. Paper presented at the ASME 2012 International Design Engineering Technical Conferences and Computers and Information in Engineering Conference.

This Conference Paper is brought to you for free and open access by the STEM Education \& Professional Studies at ODU Digital Commons. It has been accepted for inclusion in STEMPS Faculty Publications by an authorized administrator of ODU Digital Commons. For more information, please contact digitalcommons@odu.edu. 


\title{
HAPTICS-AUGMENTED TRAINING SOFTWARE FOR UNDERGRADUATE ENGINEERING MECHANICS
}

\author{
Ernur Karadogan ${ }^{1}$, Robert L. Williams II ${ }^{1}$, David R. Moore ${ }^{2}$ and Tian Luo ${ }^{2}$ \\ Mechanical Engineering Dpt., Ohio University, Athens, Ohio, USA ${ }^{1}$ \\ Educational Studies, Ohio University, Athens, Ohio, USA ${ }^{2}$ \\ Email: williar4@ohio.edu
}

\begin{abstract}
This paper presents the development efforts for a set of software activities and tutorials to augment teaching and learning in standard required undergraduate engineering mechanics courses. Using these software activities, students can change parameters, predict answers, compare outcomes, interact with animations, and feel the results. The overall system aims to increase teaching and learning effectiveness by rendering the concepts compelling, fun, and engaging. The problem with current examples and homework problems is that they are flat, static, boring, and non-engaging, which may lead to student attrition and a less than full grasp of fundamental principles. We implement integration of haptics technology with educational products to enable improvement in undergraduate engineering mechanics education. The current system is composed of a computer (laptop or desktop), a haptic device and a set of haptic modules. Currently, two modules, Interactive Free-Body Diagram (Box Motion) and Rigid Body Dynamics (Box Motion), were developed and several others are under development.
\end{abstract}

\section{KEYWORDS}

Haptics, undergraduate engineering, engineering mechanics courses, virtual reality, force feedback

\section{INTRODUCTION}

Haptics is related to the sense of touch and forces in humans. Haptic interfaces provide force and touch feedback from virtual models on the computer to human users. Existing papers relating haptics and education are largely from the medical training field. The Interventional Cardiology Training Simulator [1] linked technical simulation with specific medical education content. A virtual reality based simulator prototype for the diagnosis of prostate cancer was developed [2]. The Immersion corporation (www.immersion.com) developed haptic interfaces for injection training and sinus surgery simulation; these interfaces are expensive and special-purpose. The GROPE Project [3] developed over 30 years a 6D haptic/VR simulation of molecular docking. Howell et al. [4] describe a virtual haptic back model for improving the learning of palpatory diagnosis by medical students. A research group at the Ohio Supercomputing Center applied haptics in virtual environments to improve tractor safety by training young rural drivers [5]. Their results show haptics increases training effectiveness, but access to their unique training system is limited. Haptics was applied to make virtual environments accessible to blind persons [6,7]. The effectiveness of virtual reality in the learning process has been demonstrated by many authors [8].

Jones et al. [9] explored viruses with middle and high school students with haptic feedback from the very expensive PHANToM haptic interface. Williams et al. [10,11] developed haptics-augmented software activities and tutorials for improving the teaching and learning of K-12 science. This work included alpha and beta software testing with students. Immersion Corporation [12] investigated the potential benefits of incorporating their commercial haptic mouse into software intended for college and high school physics curricula. Bussell [13] posed the question "Can haptic technology be applied to educational software and Web sites to enhance learning and software usability?" and presented a review article. The thesis of Dede et al. [14] was that "learning difficult, abstract material can be strongly enhanced by multi-sensory immersion". Okamura et al. [15] developed their own single axis force- 
feedback 'haptic paddle' which students build to support linear systems in engineering education. Minogue and Jones [16] present a baseline review article concerning the role of touch in cognition and learning. Richard et al. [17] present a multimodal virtual environment with a range of haptic feedback, for students to explore the energy levels in the electron bound state in the Bohr atom model. Grow et al. [18] review their work in educational haptics at all levels to encourage young students to consider STEM careers. Brandt and Colton [19] investigate the suitability of the LEGO MindStorms kit for college and precollege students to build haptic interfaces to learn programming and engineering concepts.

This paper presents the overall structure of the system along with the development process for two of the haptic modules for undergraduate dynamics course: Interactive FreeBody Diagram (Box Motion) and Rigid Body Dynamics (Box Motion).

\section{SYSTEM DESCRIPTION}

\subsection{Haptic Interface}

The haptic interface for this system was chosen to be the Logitech Force ${ }^{\mathrm{TM}}$ 3D Pro Joystick (Figure 1) due to its programmable force feedback features and low cost. The system, however, can be used with any other joystick that is readily available and DirectX compatible, e.g. Microsoft Sidewinder Force Feedback joystick.

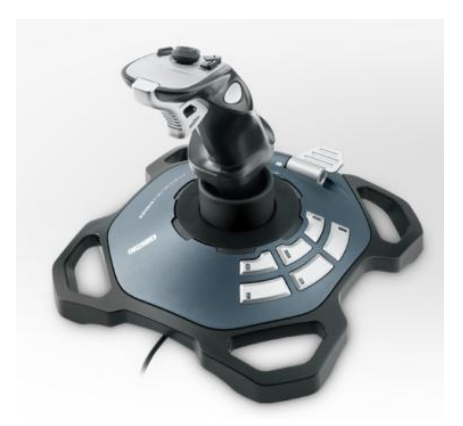

Figure 1. Logitech Force ${ }^{\mathrm{TM}}$ 3D Pro Joystick

\subsection{Process Flow}

The overall process chart is shown in Figure 2.Users must enter their user name and password in order to access the system. In the case of first time users, a new user account can be created using the sign-in menu. Once the students sign in to the system, first time users are required to view an introductory Flash tutorial before they can start their practice. This tutorial is also accessible by all users anytime during their practice via the drop-down menu at the top section of the screen. Returning users can directly sign in using their existing user name/password combination. The users can select any available module for practice and construct a new problem by changing the variables. The flexibility of changing the problem variables allows students to experiment, visualize and "feel" the corresponding changes on the forces acting on an object and its motion (when applicable) For instance, a box on the ramp may remain at rest, move up or down the ramp depending on several physical properties (mass, static friction coefficient etc.) and the amount of applied force. Once the variables are chosen, the problem is activated. At this point, the variables cannot be changed until the user manually stops or cancels the current simulation. The simulation starts by pressing the designated (trigger) button on the joystick. A significant contribution of this system is the augmentation of haptics by enabling the students to select and feel any force that is acting on the object of interest by using the joystick. The forces reflected to the users are normalized so that the maximum force value corresponds to the maximum force that the joystick can exert to user's hand.

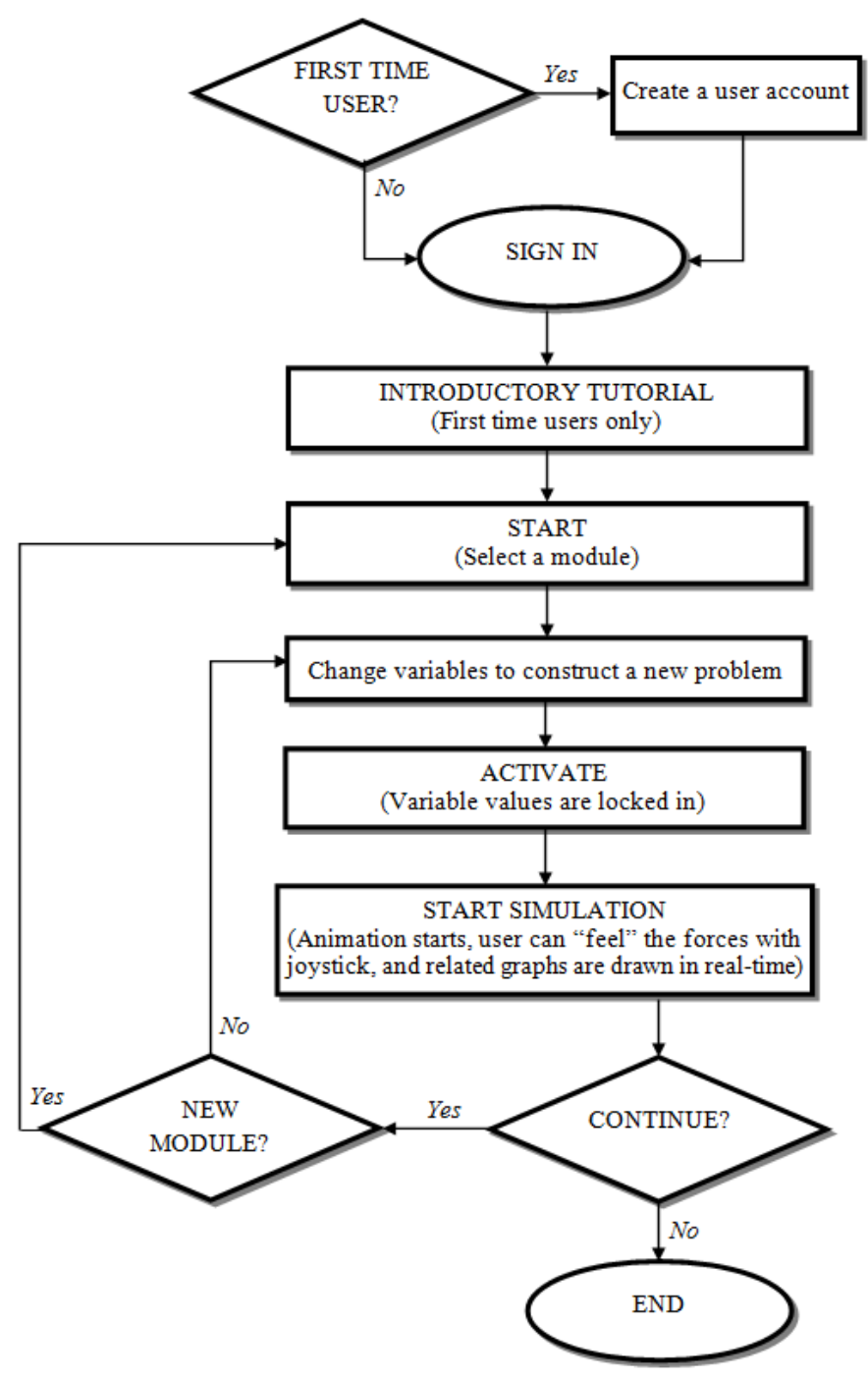

Figure 2. Process flowchart

\subsection{User Interface and Virtual Environment}

The virtual environment was designed using the same functional elements in order to ease the transition from one 
module to the other. The sign-in menu for the modules is a dialog box and serves as the entrance to the system. Using the sign-in menu, users can: 1) Create a user name and a password before they start their practice, 2) Retrieve their user name/password. Users are required to sign in to be able to access the available modules. A unique user name is necessary to store individual user data in the database to keep track of users' progress with practice.

The screen layout (Figure 3 ) is the same for all modules and consists of several elements. The simulation view is located in the middle of the screen and is the largest element for easy viewing of the simulated objects. "Course View" (Figure 4) that is on the left side of the screen allows students to select a particular module to practice or to switch another one at any time. On the right side of the screen, "Variables View" (Figure 5a) includes the variables specific to the active module. "Results and Messages View" at the bottom of the screen displays any messages, errors (e.g., if the user enters a value that is out of the acceptable range) and summary of the variables that were locked in after activating a problem. "Graphs View" (Figure 5b) appears when a problem's variables are adjusted and activated. It replaces the Course View on the screen until the simulation is stopped by the user.

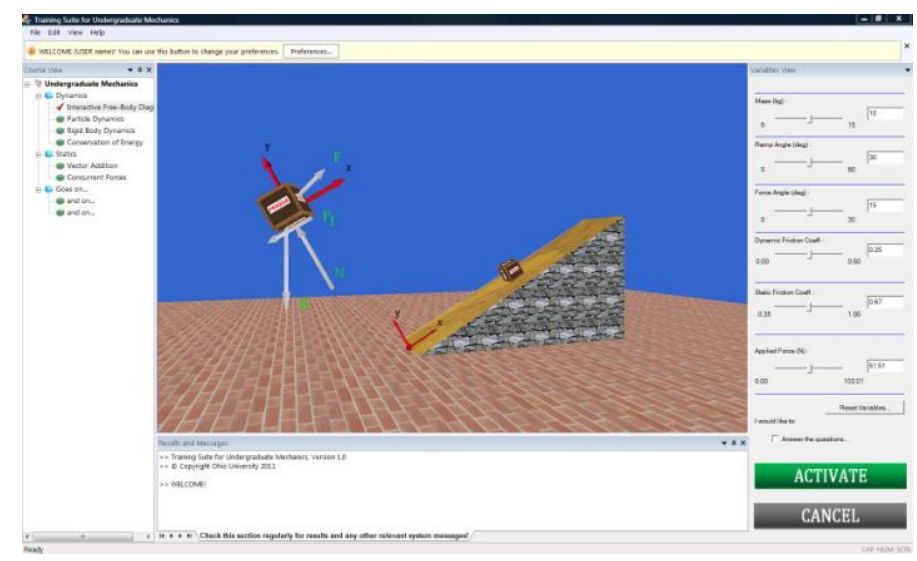

Figure 3. Screen Shot (Interactive FBD module shown)

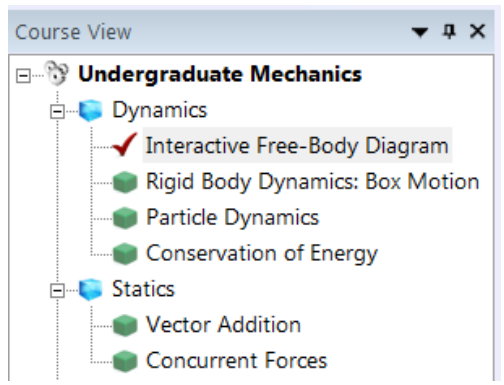

Figure 4. Course View

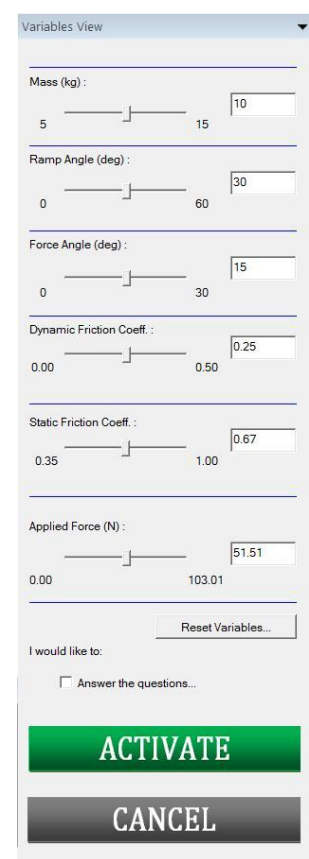

(a)

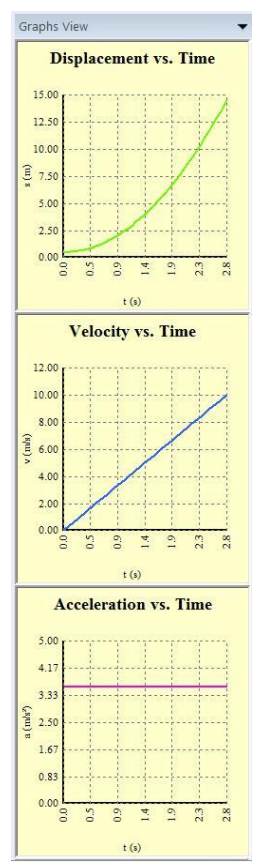

(b)
Figure 5. (a) Variables View, and (b) Graphs View

\section{MODULES DESCRIPTION}

This section presents in detail our development and implementation of the haptics-augmented Interactive FreeBody Diagram and Rigid Body Dynamics modules for undergraduate engineering dynamics. The same process and computer implementation will be followed for all ensuing haptic modules to be developed.

\subsection{Interactive Free-Body Diagram (Box Motion)}

\subsubsection{Problem Description}

A point mass $m$ is on a ramp inclined by angle $\theta$, with an applied force $F$ inclined by angle $\phi$ relative to the ramp, as shown in Figure 6 . With the point mass assumption, there can be no box rotation by definition. The static coefficient of friction between the point mass and ramp is $\mu_{s}$, and the kinetic (dynamic) coefficient of friction between the point mass and ramp is $\mu_{k}$. Figure 7 shows four available levels and their specifications for this module.

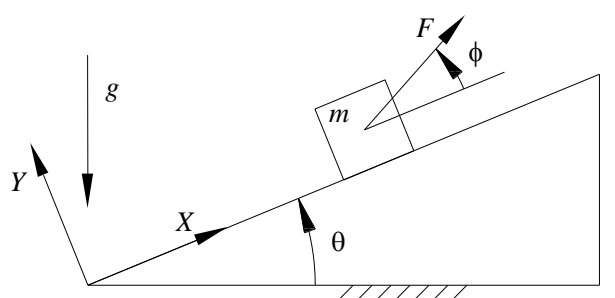

Figure 6. Interactive FBD Schematic 


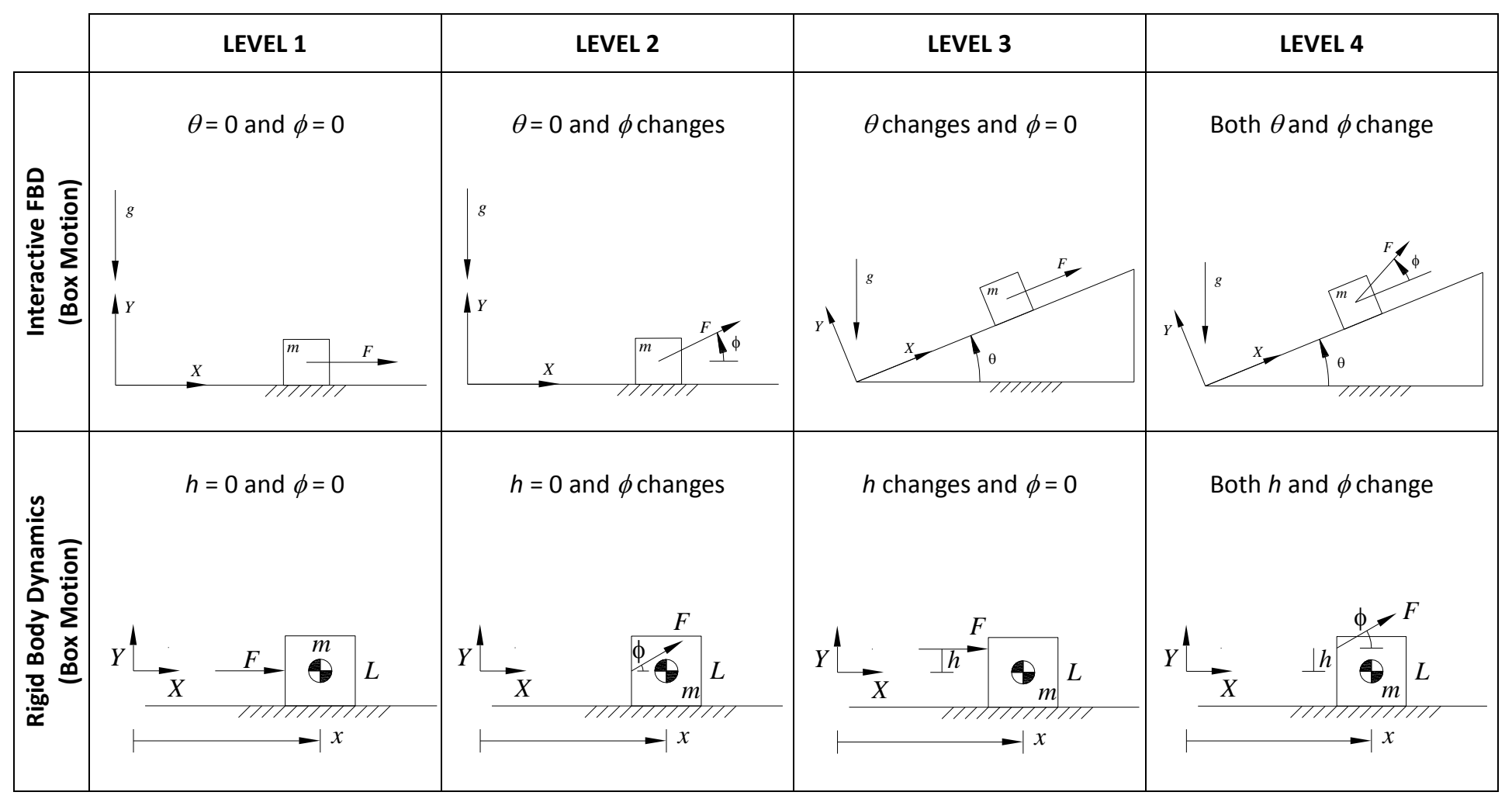

Figure 7. Level Specifications for Interactive FBD and Rigid Body Dynamics Modules

\subsubsection{Mathematical Model}

In this situation we have three distinct possibilities: the box (point mass) accelerates down the ramp, the box accelerates up the ramp, or the box is static, neither moving down nor up the ramp. The classical dynamics solution to this problem involves analyzing two cases, motion impending down the ramp and motion impending up the ramp. These two situations represent the case where motion is just about to start down (or up) the ramp. We can calculate minimum forces for these conditions, from which three cases are identified (motion down, motion up, or no motion) depending on the relative value of the applied force to all other parameters chosen by the user.

\section{Impending Motion Up the Ramp}

The free-body diagram (FBD) for the point mass box in this case is shown in Figure 8.

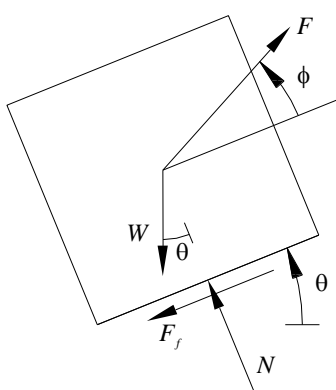

Figure 8. Interactive Free-Body Diagram, Motion Impending Up
From the free-body diagram, we apply Newton's Second Law, $\sum \mathbf{F}=m \mathbf{a}$. We use the $X Y$ coordinate system where $X$ is along the ramp and $Y$ is normal to the ramp. For the impending motion (up) case, the acceleration along the ramp is zero, $a_{x}=a=0$, and the acceleration normal to the ramp is always zero, $a_{y}=0$, assuming the point mass maintains contact to the ramp. Thus, Newton's Second Law for this case yields:

$$
\begin{aligned}
X: \sum F_{x} & =F_{\min } \cos \phi-F_{f}-W \sin \theta=m a_{x}=0 \\
Y: \sum F_{y} & =F_{\min } \sin \phi+N-W \cos \theta=m a_{y}=0
\end{aligned}
$$

Where $F_{\min }$ is the minimum force to just maintain static equilibrium (to just keep $a_{x}=a=0$ ). The static friction force in this case is $F_{f}=\mu_{s} N$ and so Eqn. (1) becomes:

$$
\begin{array}{r}
F_{\text {min }} \cos \phi-\mu_{s} N-W \sin \theta=0 \\
F_{\text {min }} \sin \phi+N-W \cos \theta=0
\end{array}
$$

The friction force $F_{f}$ must oppose the impending direction of motion as shown in the free-body diagram; $N$ is the normal force of the ramp acting on the point mass. The weight force is $W=m g$. Equation (2b) can be solved for $N$, which can be substituted into Eqn. (2a) to solve for $F_{m i n}$.

$$
F_{\text {min }}=F_{\text {min }_{u p}}=\frac{m g\left(\sin \theta+\mu_{s} \cos \theta\right)}{\cos \phi+\mu_{s} \sin \phi}
$$


We call this minimum force $F_{\min _{u p}}$ because it is the minimum applied force to maintain static equilibrium, i.e. just before acceleration is possible up the ramp.

\section{Impending Motion Down the Ramp}

The free-body diagram (FBD) for the point mass box in this case is shown in Figure 9.

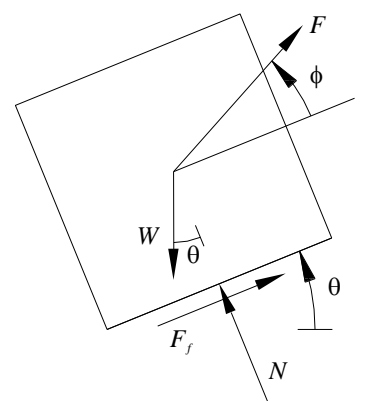

\section{Figure 9. Interactive Free-Body Diagram, Motion Impending Down}

In the $X Y$ coordinate system shown ( $X$ is aligned with the ramp, $Y$ is perpendicular to the ramp), these four force vectors are expressed as:

$$
\begin{array}{ll}
\mathbf{W}=W\left\{\begin{array}{c}
-\sin \theta \\
-\cos \theta
\end{array}\right\} & \mathbf{F}=\left\{\begin{array}{c}
F \cos \phi \\
F \sin \phi
\end{array}\right\} \\
\mathbf{N}=\left\{\begin{array}{c}
0 \\
N
\end{array}\right\} & \mathbf{F}_{\mathbf{f}}=\left\{\begin{array}{c}
F_{f} \\
0
\end{array}\right\}
\end{array}
$$

From the free-body diagram, we apply Newton's Second Law, $\sum \mathbf{F}=m \mathbf{a}$. It is convenient to use the $X Y$ coordinate system shown in the first diagram above: $X$ is along the ramp direction and $Y$ is normal to the ramp. However, for the impending motion (down) case, the acceleration along the ramp is zero, $a_{x}=a=0$, and the acceleration normal to the ramp is always zero, $a_{y}=0$, assuming the point mass maintains contact to the ramp. Thus, Newton's Second Law yields:

$$
\begin{aligned}
& X: \sum F_{x}=F_{\min } \cos \phi+F_{f}-W \sin \theta=m a_{x}=0 \\
& Y: \sum F_{y}=F_{\min } \sin \phi+N-W \cos \theta=m a_{y}=0
\end{aligned}
$$

Where $F_{\min }$ is the minimum force to just maintain static equilibrium (to just keep $a_{x}=a=0$ ). The static friction force in this case is $F_{f}=\mu_{s} N$ and so our two equations become:

$$
\begin{array}{r}
F_{\min } \cos \phi+\mu_{s} N-W \sin \theta=0 \\
F_{\min } \sin \phi+N-W \cos \theta=0
\end{array}
$$

The friction force $F_{f}$ must oppose the impending direction of motion as shown in the free-body diagram; $N$ is the normal force of the ramp acting on the point mass. The weight force is
$W=m g$. Equation (5b) can be solved for $N$, which can be substituted into Eqn. (5a) to solve for $F_{\text {min }}$ :

$$
F_{\text {min }}=F_{\text {min }_{\text {down }}}=\frac{m g\left(\sin \theta-\mu_{s} \cos \theta\right)}{\cos \phi-\mu_{s} \sin \phi}
$$

We call this minimum force $F_{\text {min }_{\text {down }}}$ because it is the minimum applied force to maintain static equilibrium, i.e. just before acceleration is possible down the ramp.

Now we have three cases to consider, distinguished by the magnitude of the applied force $F$ relative to the abovedetermined minimum forces $F_{\min _{u p}}$ and $F_{\min _{\text {down }}}$ for impending motion up and down the ramp, respectively.

\section{Case I. Box Accelerates up the Ramp when $F>F_{\min _{u p}}$}

The same equations from Newton's Second Law apply as before, but now the $X$ acceleration is non-zero, $a_{x}=a \neq 0$. The same FBD applies from the motion impending up the ramp. The equations are:

$$
\begin{aligned}
& \sum F_{x}=F \cos \phi-F_{f}-W \sin \theta=m a_{x} \\
& \sum F_{y}=F \sin \phi+N-W \cos \theta=m a_{y}=0
\end{aligned}
$$

The kinetic friction force in this case is $F_{f}=\mu_{k} N$ and so our two equations become:

$$
\begin{gathered}
F \cos \phi-\mu_{k} N-W \sin \theta=m a \\
F \sin \phi+N-W \cos \theta=0
\end{gathered}
$$

The friction force $F_{f}$ must oppose the direction of motion as shown in the free-body diagram; $N$ is the normal force of the ramp acting on the point mass. The weight force is $W=m g$. Equation (8b) can be solved for $N$, which can be substituted into Eqn. (8a) to solve for the acceleration $a$ up the ramp given the applied force $F$ :

$$
a=\frac{F\left(\cos \phi+\mu_{k} \sin \phi\right)-m g\left(\sin \theta+\mu_{k} \cos \theta\right)}{m}
$$

The box will accelerate up the ramp with $a$ in this Case I.

Case II. Box in Static Equilibrium on the Ramp when $F_{\text {min }_{\text {down }}} \leq F \leq F_{\text {min }_{u p}}$

In this case there will be no motion either up or down the ramp.

\section{Case III. Box Accelerates down the Ramp when $F<F_{\text {min }_{\text {down }}}$}

The same equations from Newton's Second Law apply as before, but now the $X$ acceleration is non-zero, $a_{x}=a \neq 0$. The same FBD applies from the motion impending up the ramp. The equations are: 


$$
\begin{aligned}
& \sum F_{x}=F \cos \phi+F_{f}-W \sin \theta=m a_{x} \\
& \sum F_{y}=F \sin \phi+N-W \cos \theta=m a_{y}=0
\end{aligned}
$$

The kinetic friction force in this case is $F_{f}=\mu_{k} N$ and so our two equations become:

$$
\begin{gathered}
F \cos \phi+\mu_{k} N-W \sin \theta=m a \\
F \sin \phi+N-W \cos \theta=0
\end{gathered}
$$

The friction force $F_{f}$ must oppose the direction of motion as shown in the free-body diagram; $N$ is the normal force of the ramp acting on the point mass. The weight force is $W=m g$. Equation (11b) can be solved for $N$, which can be substituted into Eqn. (11a) to solve for the acceleration $a$ up the ramp given the applied force $F$ :

$$
a=\frac{F\left(-\cos \phi+\mu_{k} \sin \phi\right)+m g\left(\sin \theta-\mu_{k} \cos \theta\right)}{m}
$$

The box will accelerate down the ramp with $a$ in this Case III. The negative sign of $a$ was taken into account in the above result.

\subsubsection{Implementation}

$$
\begin{aligned}
& 5 \leq m \leq 10 \\
& 0 \leq \mu_{k} \leq 0.5
\end{aligned}
$$

User sets: $m, \mu_{s}, \mu_{k}, \theta, F$, and $\phi \quad \mu_{k}<\mu_{s} \leq 1$

$$
\begin{aligned}
& 0 \leq \theta \leq 60^{\circ} \\
& 0 \leq \varphi \leq 30^{\circ}
\end{aligned}
$$

Based on these values, the computer can calculate the minimum force $F_{\text {min }_{u p}}$ and suggest the user enter a larger value for $F$.

Computer sets: $g=9.81 \mathrm{~m} / \mathrm{s}^{2}$, (down, not in the $-Y$ direction unless $\theta=0$ ).

Visualize: Free-body diagram with forces to scale, plus kinematics plots for $a, v, x$.

User Feels: Forces $F, N, F_{f}$, or $W$ (user chooses). The joystick displays the vector forces (one at a time) to the user's hand.

\subsection{Rigid Body Dynamics (Box Motion)}

\subsubsection{Problem Description}

A rigid-body box of mass $m$ is pushed along a flat motion surface by a force $F$, angled at $\phi$, as shown in the diagram below. The static and dynamic (kinetic) coefficients of friction between the box and motion surface are $\mu_{S}$ and $\mu_{K}$, respectively. The planar size of the box is a square of side $L$, the center of mass (CG) of the box is in the geometric center of the square, and force $F$ is applied a distance $h$ above the center of mass as shown in Figure 10. Figure 7 shows available levels and their specifications for this module.

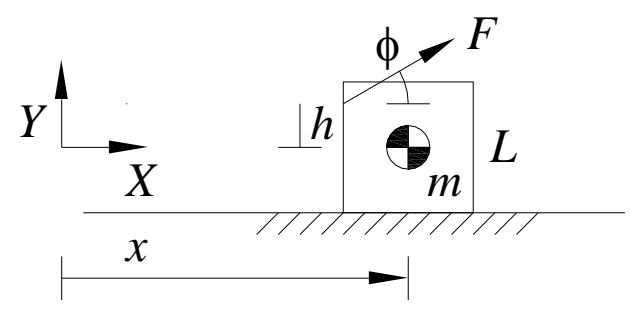

Figure 10. Rigid Body Dynamics Schematic

\subsubsection{Mathematical Model}

Since the box is considered to be a rigid body, rotation of the box must be considered in addition to translational dynamics. We must determine if the box tips as it is being translated along the motion surface. The free-body diagram (FBD) for the rigid-body box is shown in Figure 11.

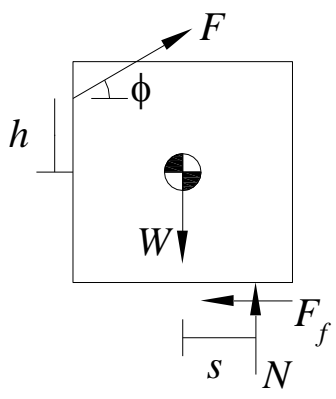

Figure 11. Free-Body Diagram for the Rigid-Body Box

\section{Static Case}

First let us calculate the applied force $F$ for impending motion. In this case the friction force is $F_{f}=\mu_{S} N$, opposing the direction of motion as shown in the FBD, where $N$ is the normal force of the motion surface acting on the box. The distance $s$ where the normal force $N$ acts is unknown. The three scalar planar equations of static equilibrium are:

$$
\begin{aligned}
\sum F_{x} & =F \cos \phi-F_{f}=0 \\
\sum F_{y} & =N-W+F \sin \phi=0 \\
\sum M_{C G z} & =N s-F \frac{L}{2} \sin \phi-F h \cos \phi-F_{f} \frac{L}{2}=0
\end{aligned}
$$

Using Eqns. (13a) and (13b), we can solve for the applied force for impending motion:

$$
F_{i m p}=\frac{\mu_{S} m g}{\cos \phi+\mu_{S} \sin \phi}
$$

where the weight is $W=m g$.

Now let us consider the case of impending tipping (rotation) of the box, prior to the impending translation motion. In this case the friction force is unknown, while the distance where the normal force $N$ acts is on the corner of the box, $s=$ $L / 2$. The previous three equations of static equilibrium (Eqns. (13)) still apply; substituting Eqns. (13a) and (13b) into (13c) yields the impending tipping force: 


$$
F_{t i p}=\frac{m g L}{2\left[L \sin \phi+\left(h+\frac{L}{2}\right) \cos \phi\right]}
$$

Now, we must have $F_{\text {imp }}<F_{\text {tip }}$ if the box is to accelerate to the right without tipping over around the lower-right corner of the box. Thus:

$$
\begin{aligned}
F_{\text {imp }} & <F_{\text {tip }} \\
\frac{\mu_{S} m g}{\cos \phi+\mu_{S} \sin \phi} & <\frac{m g L}{2\left[L \sin \phi+\left(h+\frac{L}{2}\right) \cos \phi\right]}
\end{aligned}
$$

Simplifying the above inequality, we find the following condition to ensure impending translational motion to the right without tipping:

$$
\phi<\tan ^{-1}\left[\frac{L-2 \mu_{S}\left(h+\frac{L}{2}\right)}{\mu_{S} L}\right]=\tan ^{-1}\left[\frac{1}{\mu_{S}}-\frac{2 h}{L}-1\right]
$$

\section{Dynamic Case}

First let us calculate the minimum force $F$ for dynamic motion. In this case the friction force is $F_{f}=\mu_{K} N$ and the distance $s$ where the normal force $N$ acts is unknown. The three scalar planar dynamic equations of motion are:

$$
\begin{aligned}
\sum F_{x} & =F \cos \phi-F_{f}=m a_{x} \\
\sum F_{y} & =N-W+F \sin \phi=m a_{y}=0 \\
\sum M_{C G z} & =N s-F \frac{L}{2} \sin \phi-F h \cos \phi-F_{f} \frac{L}{2}=I_{G Z} \alpha=0
\end{aligned}
$$

where we have assumed that the box slides to the right without tipping (no rotation) so that the $Y$ component of the translational acceleration and the rotational acceleration are both zero.

Using Eqns. (17a) and (17b), we can solve for the $X$ acceleration $a_{x}$ :

$$
a_{x}=\frac{1}{m}\left[F \cos \phi+F \mu_{K} \sin \phi-\mu_{K} m g\right]
$$

from which we can calculate the minimum applied force for dynamic motion (taking $a_{x}=0$ ):

$$
F_{\min }=\frac{\mu_{K} m g}{\cos \phi+\mu_{K} \sin \phi}
$$

From Eqn. (17c), again assuming no tipping, the distance $s$ from the $\mathrm{CG}$ where the normal force $N$ acts is:

$$
s=\frac{F L \sin \phi\left(1-\mu_{K}\right)+2 F h \cos \phi+\mu_{K} m g L}{2(m g-F \sin \phi)}
$$

Now let us consider the onset of dynamic tipping of the box. In this case the friction force is unknown, while the distance where the normal force $N$ acts is on the corner of the box, $s=L / 2$, and the angular acceleration of the box is still just remaining zero. The previous three dynamic equations (Eqns. (17)) of motion still apply; Eqn. (17b) into Eqn. (17c) with $\alpha=$ 0 and $s=L / 2$ yields the friction force:

$$
F_{f}=m g-2 F \sin \phi-\frac{2 F h \cos \phi}{L}
$$

Tipping occurs when the maximum dynamic friction $\mu_{K} N$ is greater than or equal to the above-calculated friction force $F_{f}$. For no tipping we have $F_{f}>\mu_{K} N$ which yields the maximum applied dynamic force for no tipping:

$$
F_{\text {max }}=\frac{m g\left(1-\mu_{K}\right)}{\left(2-\mu_{K}\right) \sin \phi+2 \frac{h}{L} \cos \phi}
$$

\subsubsection{Implementation}

$$
\begin{array}{ll} 
& 0 \leq h \leq L / 2 \\
\text { User sets: } h, \mu_{K}, \phi & 0 \leq \mu_{k} \leq 0.4 \\
& \mu_{k}<\mu_{s} \leq 1
\end{array}
$$

Based on these values (plus $m, g$, and $L$ set by the computer below), the computer can calculate the bounds on applied force $F$ as discussed above and suggest the user enter an appropriate value for $F: F_{\min } \leq F \leq F_{\max }$ to ensure dynamic motion to the right without tipping.

Computer sets: $\quad g=9.81 \mathrm{~m} / \mathrm{s}^{2}$, (in the $-Y$ direction), $L=1 \mathrm{~m}$.

Visualize: Box motion to the right, plus kinematics plots for $a$, $v, x$.

User Feels: Forces $F, N, F_{f}$, or $W$ (user chooses). The joystick displays the vector forces (one at a time) to the user's hand.

\section{CONCLUSION}

In this paper, the haptic-augmented training software to increase teaching and learning effectiveness of undergraduate mechanics courses was introduced. The development processes of two haptic modules (Interactive Free-Body Diagram and Rigid Body Dynamics) were described in detail. This system has potential to increase engagement of undergraduate engineering students by providing real-time animation of physical systems in a virtual environment and by feeling relative magnitude of the forces acting on the objects of interest by means of a haptic device. The completed modules are currently under evaluation with the participation of undergraduate engineering students.

\section{REFERENCES}

[1] Shaffer D., Meglan D., Ferrell M., and S. Dawson, 1999, "Virtual Rounds: Simulation-Based Education in Procedural Medicine", Proceedings of the 1999 SPIE 
Battlefield Biomedical Technologies Conference, Orlando, FL, 3712: 99-108.

[2] Burdea G., Patounakis G., and Popescu V., 1999, "Virtual Reality-Based Training for the Diagnosis of Prostate Cancer", IEEE Transactions on Biomedical Engineering, 46(10): 1253-60.

[3] Brooks Jr. F.P., Ming O.-Y., Batter J.J., and Kilpatrick P.J., 1990, "Project GROPE: Haptic Displays for Scientific Visualization", Computer Graphics (ACM), 24(4): 177 185.

[4] Howell J.N., Conatser Jr. R.R.,. Williams II R.L, Burns J.M., and Eland D.C., 2008, "The Virtual Haptic Back: A Simulation for Training in Palpatory Diagnosis", BMC Medical Education, April: 8-14.

[5] Stredney D., Wiet G.J., Yagel R., Sessanna D., Kurzion Y., Fontana M., Shareef N., Levin M., Martin K., and Okamura A., 1998, "A Comparative Analysis of Integrating Visual Representations with Haptic Displays", Proceedings of MMVR6, Westwood et al., Editors, IOS Press, Amsterdam: 20-26.

[6] Treviranus J., 2000, "Adding Haptics and Sound to Spatial Curriculum", Proc IEEE Intl Conference on Systems, Man and Cybernetics, Nashville, TN, October 8-11, 1: 588-592.

[7] Jansson G., Petrie H., Colwell C., Kornbrot D., Fänger J., König H., Billberger K., Hardwick A., and Furner S., 1999, "Haptic Virtual Environments for Blind People: Exploratory Experiments with Two Devices", International Journal of Virtual Reality, 4(1).

[8] North S.M., 1996, "Effectiveness of Virtual Reality in the Motivational Processes of Learners", International Journal of Virtual Reality, 2(1).

[9] Jones M.G., Bokinsky A., Andre T., Kubasko D., Negishi A., Taylor R., and Superfine R., 2002, "NanoManipulator Applications in Education: The Impact of Haptic Experiences on Students' Attitudes and Concepts", Proceedings of the IEEE Computer Science Haptics Symposium, Orlando, FL: 295-298.
[10] Williams II R.L., Chen M.Y., and Seaton J.M., 2003, "Haptics-Augmented Simple Machines Educational Tutorials", Journal of Science Education and Technology, 12(1): 16-27.

[11] Williams II R.L., Chen M.Y., and Seaton J.M., 2002, "Haptics-Augmented High School Physics Tutorials", International Journal of Virtual Reality, 5(1).

[12] Wies E.F., Gardner J.A., O'Modhrain M.S., Hasser C.J., and Bulatov V.L., 2001, "Web-based Touch Display for Accessible Science Education", Haptic Human-Computer Interaction: First International Workshop, Glasgow, UK, 2058: 52-60.

[13] Bussell L., 2001, "Haptic Interfaces: Getting in Touch with Web-based Learning”, Journal of Educational Technology, 41(3): 27-32.

[14]Dede C., Salzman M.C., and Loftin R.B., 1996, "ScienceSpace: Research on using Virtual Reality to enhance Science Education", World Conference on Educational Multimedia and Hypermedia, Boston, MA, June 17-22: 172-177.

[15] Okamura A.M., Richard C., and Cutkosky M.R., 2002, "Feeling is Believing: Using a Force-Feedback Joystick to Teach Dynamic Systems", Jnl of Engineering Education, 91(3): 345-349.

[16] Minogue J., and Jones M.G., 2006, "Haptics in Education: Exploring an Untapped Sensory Modality", Review of Educational Research, 76(3): 317-348.

[17] Richard E., Tijou A., and Richard P., 2006, "Multi-modal virtual environments for education: From illusion to immersion", Technologies for e-Learning and Digital Entertainment, 3942: 1274-1279.

[18] Grow D.I., Verner L.N., and Okamura A.M., 2007, "Educational Haptics", AAAI Spring Symposium, v SS07-09: 53-58.

[19]Brandt A.M., and Colton M.B., 2008, "Toys in the Classroom: LEGO MindStorms as an Educational Haptics Platform", IEEE Symposium on Haptics Interfaces for Virtual Environment and Teleoperator Systems: 389-395. 